\title{
$N$-氟代苯磺酰亚胺参与的过渡金属催化 $\mathrm{C}-\mathrm{H}$ 氟化和胺化的研究进展
}

\author{
王威霖 $a$ 陈卫东 $a$ 骆钧飞*, $a$ 解 攀*, $b$ \\ ( ${ }^{a}$ 宁波大学材料科学与化学工程学院 浙江宁波 315211) \\ ( ${ }^{b}$ 陕西科技大学化学与化工学院 陕西省轻化工助剂重点实验室 西安 710021)
}

\begin{abstract}
摘要 众多胺类及含氟化合物具有重要的生理活性, 在医药领域均具有不可替代的作用. 过渡金属催化的 $\mathrm{C}-\mathrm{H}$ 胺化 及氟化反应因其高反应效率及原子经济性, 受到了合成化学家的关注, 为生物碱类天然产物及含氟分子的合成提供了 便利. $N$-氟代双苯磺酰胺(NFSI) 兼有氟原子及含氮官能团, 可以在过渡金属催化下参与多种类型的有机反应, 实现 C$\mathrm{H}$ 键的氟化或胺化. 因此, 探索 NFSI 参与的 $\mathrm{C}-\mathrm{H}$ 键直接氟化或胺化反应具有重要意义. 综述了近十年 NFSI 参与的 C $-\mathrm{H}$ 活化构建 $\mathrm{C}-\mathrm{N}$ 键和 $\mathrm{C}-\mathrm{F}$ 键方法的研究进展, 围绕各类方法的反应机理和应用范围进行阐述, 同时对该领域的局 限性和发展前景进行总结和展望.
\end{abstract}

关键词 $N$-氟代苯磺酰亚胺, 氟化, 胺化, $\mathrm{C}-\mathrm{H}$ 键活化, 过渡金属催化

\section{Recent Advances in $\mathrm{C}-\mathrm{H}$ Fluorination and Amination with $\mathrm{N}$-Fluorobenzenesulfonimide}

\author{
Wang, Weilin ${ }^{a}$ \\ Chen, Weidong ${ }^{a}$ \\ Luo, Junfei*,a \\ Xie, $\operatorname{Pan}^{*, b}$ \\ ( ${ }^{a}$ School of Materials Science and Chemical Engineering, Ningbo University, Ningbo, Zhejiang 315211) \\ ( ${ }^{b}$ College of Chemistry and Chemistry Engineering, Shaanxi Key Laboratory of Chemistry Additives for Industry, \\ Shaanxi University of Science \& Technology, Xi'an 710021)
}

\begin{abstract}
The nitrogen- and fluorine-containing molecules display multiple important bioactivities which are crucial compounds in medicinal chemistry. The strategy relies on the transition-metal-catalyzed $\mathrm{C}-\mathrm{H}$ amination and fluorination has received much attention due to its atom- and step-economy, providing an alternative to the synthesis of many natural alkaloids and fluorides. $\mathrm{N}$-Fluorobenzenesulfonimide (NFSI) consists of the fluoride atom and the nitrogen-containing functionality, it is frequently used in the reactions based on transition-metal-catalyzed $\mathrm{C}-\mathrm{H}$ activation to construct both $\mathrm{C}-\mathrm{N}$ and $\mathrm{C}-\mathrm{F}$ bonds. In this mini-review, the recent research advances in the formation of $\mathrm{C}-\mathrm{N}$ and $\mathrm{C}-\mathrm{F}$ bonds through transition-metal-catalyzed $\mathrm{C}-\mathrm{H}$ with NSFI are reviewed. The reaction scopes and mechanisms are discussed in details, and the limitations of current procedures and the prospects for the future are summarized.

Keywords $N$-fluorobenzenesulfonimide, fluorination, amination, $\mathrm{C}-\mathrm{H}$ activation, transition-metal catalysis
\end{abstract}

近年来, 过渡金属催化 $\mathrm{C}-\mathrm{H}$ 键活化构建新的 $\mathrm{C}-$ $\mathrm{X}$ 键 $(\mathrm{X}$ 为 $\mathrm{C}$ 或杂原子 $)$ 的策略已成为分子官能团化或分 子多样化的强力工具 ${ }^{[1]}$, 同时也为天然产物或药物分子 后期修饰提供了便捷的途径 ${ }^{[2]}$. 目前, 较为成熟的 $\mathrm{C}-\mathrm{H}$ 键活化方法是通过过渡金属活化某一分子(A)中特定的 $\mathrm{C}-\mathrm{H}$ 键，并与另一分子 $(\mathbf{B})$ 反应，实现两个分子的偶联. 在这一过程中, 偶联分子 $\mathbf{B}$ 往往只能实现某一共价键的 构建并离去特定官能团(Scheme 1, a). 比如芳基硼酸化
合物一般通过离去嗍盐，构建 $\mathrm{C}-\mathrm{C}(\operatorname{aryl})$ 键 $^{[3]} ; N$-卤代琥 珀酰亚胺可以离去琥珀酰亚胺组分用于 $\mathrm{C}-\mathrm{X}(\mathrm{X}$ 为卤 素)键的构建 ${ }^{[4]}$.

$N$-氟代双苯磺酰胺(NFSI)作为一种温和的氟化试 剂, 其氟正离子 $\left(\mathrm{F}^{+}\right)$的存在使其参与不同类型的亲电氟 化反应 ${ }^{[5]}$; 同时具有强氧化能力的氟正离子还可将过渡 金属氧化为高价态金属化合物 ${ }^{[6]}$; NFSI 还可以作为自 由基氟化试剂，与烷基自由基作用发生氟化过程 ${ }^{[7]}$. 除

\footnotetext{
* Corresponding authors. E-mail: luojunfei@nbu.edu.cn; pan.xie@sust.edu.cn

Received June 29, 2020; revised August 11, 2020; published online September 8, 2020.

Project supported by the Ningbo Municipal Natural Science Foundation (No. 2019A610027), and the Education Foundation of Shaanxi Province (No. 18JK0105).

宁波市自然科学基金(No. 2019A610027) 和陕西省教育厅专项科研计划(No. 18JK0105)资助项目.
} 
此以外, NFSI 可以作为胺化试剂构建不同类型的 $\mathrm{C}-\mathrm{N}$ 键. 通常情况下, NFSI 可以作为亲核性或自由基胺基源 参与烯烃的双官能团化反应如双胺基化 ${ }^{[8]}$ 、胺基氟化 ${ }^{[9]}$ 、 胺基氰基化 ${ }^{[10]}$ 、胺基叠氮 ${ }^{[11]}$ 和碳胺化 ${ }^{[12]}$ 等, 另外也可以 实现芳(杂)环、茮基位和烯丙基位的胺化反应 ${ }^{[13]}$. 近年 来, 有机化学家在研究过渡金属催化 $\mathrm{C}-\mathrm{H}$ 键活化反应 的过程中发现， NFSI 不仅可以用于 $\mathrm{C}-\mathrm{H}$ 氟化反应， 同时也可以实现 $\mathrm{C}-\mathrm{H}$ 胺化反应(Scheme 2, b), 这与绝 大多数偶联试剂在 $\mathrm{C}-\mathrm{H}$ 键活化反应中只能构建单一共 价键不同. 另一方面, 氟化合物的特殊性以及胺类化合 物极重要的生理活性, 使其在医药领域中具有不可替代 的作用. 因此我们对近十年来 NFSI 作为偶联试剂参与 的过渡金属催化的 $\mathrm{C}-\mathrm{H}$ 键活化构建 $\mathrm{C}-\mathrm{F}$ 键和 $\mathrm{C}-\mathrm{N}$ 键的方法进行了总结, 相信这将对含氟和胺类化合物的 高效合成方法的发展提供一定帮助. 同时, 对 NFSI 参 与的在 $\mathrm{C}-\mathrm{H}$ 氟化及胺化反应机理进行了讨论, 这对过 渡金属催化的 $\mathrm{C}-\mathrm{H}$ 键活化方法中新型偶联试剂的开发 具有一定的参考价值.

a) $\mathrm{R}^{1}-\mathrm{H}+\mathrm{X}-\mathrm{R}^{2} \stackrel{[\mathrm{M}]}{\longrightarrow} \mathrm{R}^{1}-\mathrm{R}^{2}$

A B

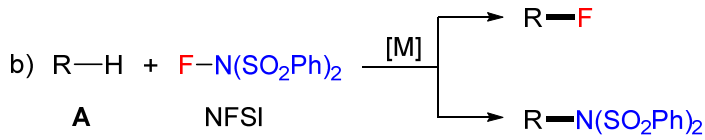

图式 1 常见 $\mathrm{C}-\mathrm{H}$ 键活化偶联试剂和 NFSI 参与的 $\mathrm{C}-\mathrm{H}$ 键 活化

Scheme $1 \mathrm{C}-\mathrm{H}$ activation with common coupling parther and $\mathrm{C}-\mathrm{H}$ activation with NFSI

\section{$1 \mathrm{NFSI}$ 参与的 C-H 氟化反应}

\section{$1.1 \mathrm{C}\left(\mathrm{sp}^{2}\right)-\mathrm{H}$ 氟化}

目前过渡金属催化选择性 $\mathrm{C}-\mathrm{H}$ 键活化主要是通过 导向基团与过渡金属配位，诱导其活化邻位 $\mathrm{C}-\mathrm{H}$ 键形 成环金属中间体(通常为五元或六元环), 比如 Scheme 2 所示的环钯化合物 2, 在 NFSI 存在下环钯化合物 $\mathbf{2}$ 发生 氧化加成得到中间体 3 , 一般情况下, 由于氟原子的强 电负性, $\mathrm{Pd}-\mathrm{F}$ 键不易发生还原消除. 但是双苯磺酰胺 基 $\left[\mathrm{N}\left(\mathrm{SO}_{2} \mathrm{Ph}\right)_{2}\right]$ 作为配体时, 其较大的空间位阻可以促进 中间体 3 还原消除过程的发生, 从而得到氟化产物.

许丹倩团队在此领域做了很大的贡献. 2013 年, 许 丹倩和徐振元等 ${ }^{[14]}$ 利用多元含氮杂环如喹喔啉、吡嗪、 苯并噁唑和吡唑等作为导向基团, 以金属钯盐作为催化 剂, NFSI 作为氟化试剂, 成功实现了杂环邻位的选择性 单氟化反应(Eq. 1). 该反应过程中, 三氟乙酸(TFA)的加 入可大幅提升产率. 如果使用 $\mathrm{Pd}(\mathrm{TFA})_{2}$ 代替 $\operatorname{Pd}(\mathrm{OAc})_{2}$, 即使不额外添加三氟乙酸, 反应收率同样可以得到提

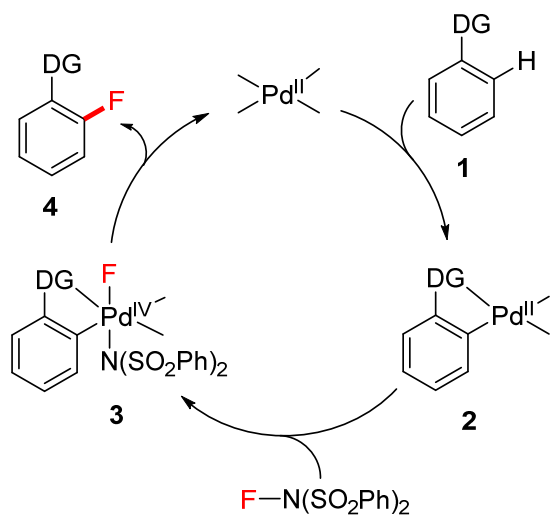

图式 2 NFSI 参与的钯催化 $\mathrm{C}-\mathrm{H}$ 氟化反应机理示意图 Scheme 2 Catalytic cycle for Pd-catalyzed $\mathrm{C}-\mathrm{H}$ fluorination with NFSI

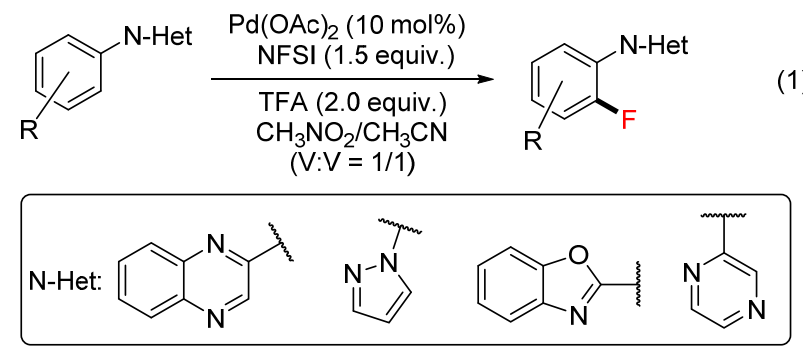

升. 作者推测可能的原因是碳氢活化步骤释放的催化量 三氟乙酸促进了催化循环的完成. 机理研究表明反应经 历了 $\operatorname{Pd}(\mathrm{II}) / \mathrm{Pd}(\mathrm{IV})$ 的催化循环过程.

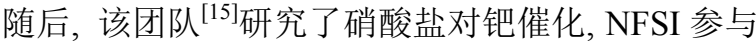
的 $\mathrm{C}\left(\mathrm{sp}^{2}\right)-\mathrm{H}$ 氟化反应的促进作用(Eq. 2). 作者发现不 同的硝酸盐均可以促进反应的进行，其中硝酸钾表现出 最好的效果. $\mathrm{Pd}\left(\mathrm{NO}_{3}\right)_{2} \cdot 2 \mathrm{H}_{2} \mathrm{O}$ 作为反应催化剂时可得到 与 $\mathrm{Pd}_{2}(\mathrm{dba})_{3}-\mathrm{KNO}_{3}$ 催化体系类似的结果, 因此作者推断 阳离子钯物种 $\left[\mathrm{Pd}\left(\mathrm{NO}_{3}\right)\right]^{+}$可能更容易活化 $\mathrm{C}-\mathrm{H}$ 键, 另 外弱亲核性的硝酸根基团可能对还原消除过程具有一 定的促进作用. 该催化体系由 $O$-甲基酮肜基团导向，在 室温或接近室温的条件下就能实现芳环邻位 $\mathrm{C}-\mathrm{H}$ 氟化 和烯基 $\mathrm{C}\left(\mathrm{sp}^{2}\right)-\mathrm{H}$ 键的 $\beta$ 位氟化.
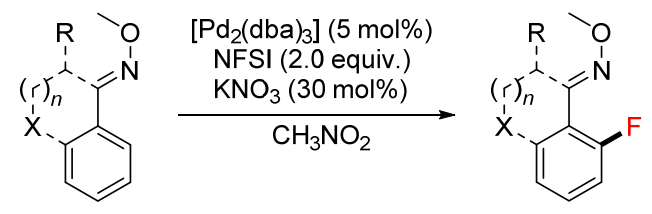

(2)

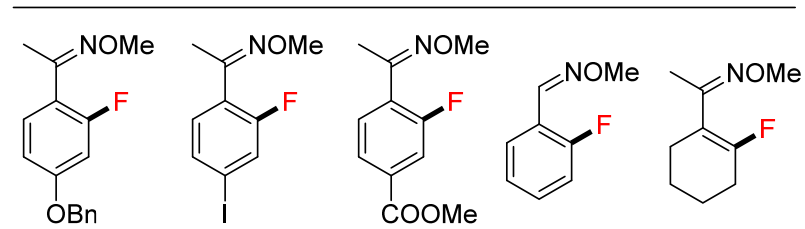

$\left(25^{\circ} \mathrm{C} ; 80 \%\right)\left(40^{\circ} \mathrm{C} ; 81 \%\right) \quad\left(40^{\circ} \mathrm{C} ; 82 \%\right)\left(70{ }^{\circ} \mathrm{C} ; 71 \%\right)\left(25^{\circ} \mathrm{C} ; 73 \%\right)$

之后，许丹倩课题将 NFSI 参与 $\mathrm{C}-\mathrm{H}$ 氟化策略运用 到其它具有重要作用的化合物的合成中. 比如该课题 
组 ${ }^{[16]}$ 以吡啶作为导向基团, 实现了钯催化苯酚邻位的 $\mathrm{C}-\mathrm{H}$ 键氟化的反应(Eq. 3). 该反应具有非常高的官能 团兼容性, 强吸电子基团和强给电子基团取代的反应底 物均具有较好的反应活性. 利用该策略, 吡氟酰草胺 (diflufenican) 以 $75 \%$ 的收率得到邻位选择性氟化产物. 另外以 NFSI 作为氟化试剂, 苯甲酰胺和苯乙酰胺亦可 在钯催化条件下实现邻位的 $\mathrm{C}-\mathrm{H}$ 氟化反应(Eq. 4) ${ }^{[17]}$. 通过该方法可快速制备含氟的镇痛药布洛芬衍生物.
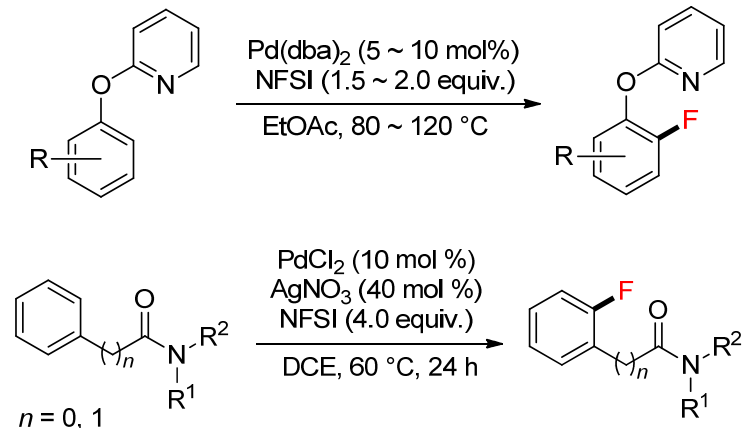

$S$-四嗪( $s$-tetrazines)具有重要的生物活性, 其特殊的 物理化学性质使其在材料能源领域扮演重要角色. 但是 $S$-四嗪容易被金属还原而分解, 因此过渡金属催化 $S$-四 嗪的 $\mathrm{C}-\mathrm{H}$ 键官能团化的反应报道相对较少. 2016 年, Rogr 和 Hierso 等 ${ }^{[18]}$ 实现了钯催化 $S$-四嗪的 $\mathrm{C}-\mathrm{H}$ 卤代 反应, 使用 $N$-氯代琥珀酰亚胺、 $N$-溴代琥珀酰亚胺和 $N$-碘代琥珀酰亚胺分别实现氯代、溴代和碘代产物的合 成. NFSI 作为卤化试剂, 反应则生成氟代产物(Eq. 5). 通过控制卤化试剂的用量, 选择性地生成单卤代或多卤 代产物.

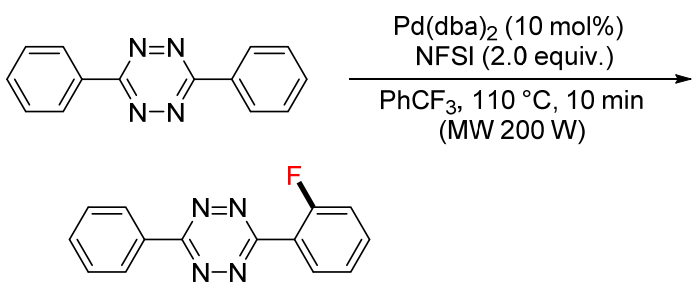

Roger, Hierso 和 Lessard 等 ${ }^{[19]}$ 研究了以芳基吡唑为 导向基团, NFSI 为氟化试剂的 $\mathrm{C}-\mathrm{H}$ 氟化反应(Eq. 6). 苯环上的取代基团对反应影响较小, 但是吡唑环上的取 代基对反应有明显的影响. 吡唑环 C5 位有取代基团时 反应被完全抑制, 这可能是由于位阻的原因, C3 位取代 基团只有是给电子基团时才能反应，且收率较低; 而 C4 位的取代基团影响较小, 不论吸电子还是给电子基 团都能得到较好的收率. 值得注意的是, 作者通过密度 泛函理论(DFT)计算发现: 不同与传统的 $\operatorname{Pd}(\mathrm{II}) / \mathrm{Pd}(\mathrm{IV})$ 催化循环, 该反应不经历 $\operatorname{Pd}(\mathrm{IV})$ 中间体, NFSI 与二价环 钯配合物作用, 经过过渡态 $\mathbf{5}$ 即可生成氟代产物. 反应
需要高浓度的 NFSI, 为该推测提供一定的依据.

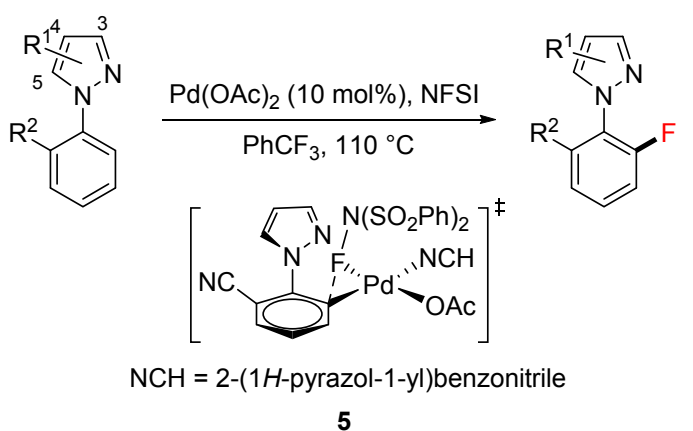

其他导向基团辅助的 $\mathrm{C}-\mathrm{H}$ 氟化反应也相继被报 道，从而丰富了 NFSI 参与的钯催化 $\mathrm{C}-\mathrm{H}$ 活化的反应体 系. 蒲守智课题组 ${ }^{[20]}$ 利用噻唑基团的导向实现了邻位 $\mathrm{C}\left(\mathrm{sp}^{2}\right)-\mathrm{H}$ 键的氟化反应( $\mathrm{Eq}$. 7). 这是首例通过 $\mathrm{C}-\mathrm{H}$ 键 官能化来实现氟代 1,3-苯并噻唑合成的反应. 作者推测 反应经历了 $\mathrm{Pd}(\mathrm{II}) / \mathrm{Pd}(\mathrm{IV})$ 的催化循环过程, 其中 $L$-脯氨 酸配体在二价钯到四价钯中间体的氧化加成过程中起 到了关键作用.

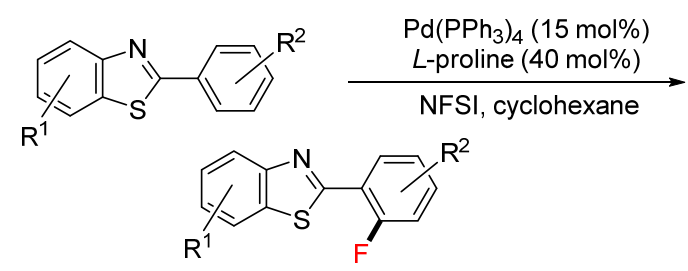

赵应声课题组 ${ }^{[21]}$ 以乙二酰胺基作为导向基团实现 了钯催化苯乙胺衍生物邻位的 $\mathrm{C}-\mathrm{H}$ 氟化反应(Eq. 8). 该导向基团可能通过双齿配位与 $\mathrm{Pd}$ 催化剂作用，得到 环钯中间体 6. 当催化剂用量提高到 $10 \mathrm{~mol} \%$ 时，反应 生成双氟取代产物. 反应氟代产物在碱性条件下即可脱 去保护基得到邻位氟代的苯乙胺类化合物.<smiles>[R]c1ccc(C([R])NC(=O)C(=O)NCc2ccccc2)cc1</smiles>
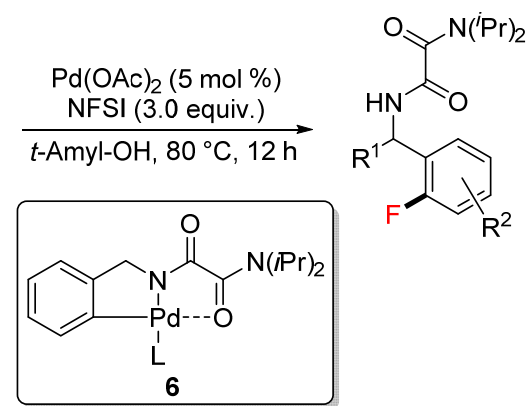

2016 年, $\mathrm{Li}$ 课题组 ${ }^{[22]}$ 利用二甲基噁唑啉的导向作 用, 以 NFSI 为氟化试剂, 在 $\operatorname{Pd}(\mathrm{II})-\mathrm{Ag}(\mathrm{I})$ 催化体系下实 现了邻位的 $\mathrm{C}\left(\mathrm{sp}^{2}\right)-\mathrm{H}$ 键氟化反应( $\left.\mathrm{Eq} .9\right)$. 反应产物的 导向基团脱除即可得到邻位氟代的苯甲酸衍生物. 另 外, Hong 课题组 ${ }^{[23]}$ 在研究 2-苯基喹唑啉-4(3H)-酮的 C- 
$\mathrm{H}$ 活化反应中发现, NFSI 也可作为氟化试剂实现苯环邻 位的氟化作用(Eq. 10). 反应经历 $\mathrm{Pd}(\mathrm{II}) / \mathrm{Pd}(\mathrm{IV})$ 催化循环 过程.
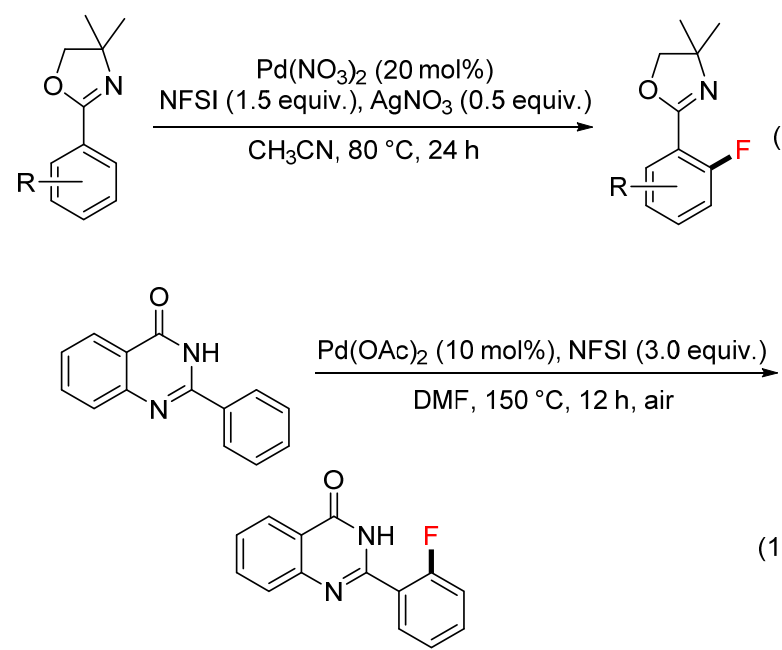

\section{$1.2 \mathrm{C}\left(\mathrm{sp}^{3}\right)-\mathrm{H}$ 氟化}

与 $\mathrm{C}\left(\mathrm{sp}^{2}\right)-\mathrm{H}$ 键相比, $\mathrm{C}\left(\mathrm{sp}^{3}\right)-\mathrm{H}$ 键的键能更大, 因 此金属催化剂催化活化 $\mathrm{C}\left(\mathrm{sp}^{3}\right)-\mathrm{H}$ 键并实现氟化反应更 具挑战性. 2015 年, 徐云根课题组 ${ }^{[24]}$ 以 8-氨基喹啉作为 双齿导向基团, NFSI 为氟化试剂, 开发了钯催化羧酸衍 生物的 $\mathrm{C}\left(\mathrm{sp}^{3}\right)-\mathrm{H}$ 氟化反应(Eq. 11). 作者发现 $\mathrm{Ag}_{2} \mathrm{O}$ 和 特戊酸是成功的关键, 使用其他金属氧化剂或酸添加剂 时, 反应效率均很低. 酸性条件下导向基团非常容易脱 除, 得到 $\beta$ 位氟代的羧酸衍生物. 反应经历 $\operatorname{Pd}(\mathrm{II}) /$ $\operatorname{Pd}(\mathrm{IV})$ 催化循环过程，双齿导向基团与钯催化剂配位， 活化 $\beta$ 位 $\mathrm{C}\left(\mathrm{sp}^{3}\right)-\mathrm{H}$ 键, 其与 NFSI 发生氧化加成可得到 四价钯中间体 $\mathrm{X}$, 进一步还原消除即可得到 $\beta$ 位氟代产 物.<smiles>[R]CCC(=O)Nc1cccc2cccnc12</smiles>

$\mathrm{Pd}(\mathrm{OAc})_{2}(15 \mathrm{~mol} \%)$ $\stackrel{\mathrm{NFSI}(2.0 \text { equiv. }), \mathrm{Ag}_{2} \mathrm{O}(1.0 \text { equiv. })}{\mathrm{PivOH}(1.0 \text { equiv. }), \mathrm{PhCl}, 90 \sim 120^{\circ} \mathrm{C}}$<smiles>[R]C(F)CC(=O)Nc1cccc2cccnc12</smiles>

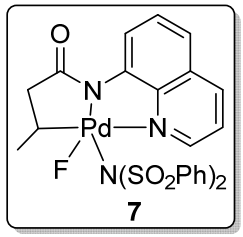

2018 年, 利用外导向基团的协助, 许丹倩和娄绍杰 等 ${ }^{[25]}$ 报道了钯催化醇衍生物的 $\mathrm{C}-\mathrm{H}$ 键 (芳香族 $\mathrm{C}-\mathrm{H}$ 键、甲基 $\mathrm{C}-\mathrm{H}$ 键、亚甲基 $\mathrm{C}-\mathrm{H}$ 键以及茮型 $\mathrm{C}-\mathrm{H}$ 键) 选择性氟化反应. $\mathrm{C}\left(\mathrm{sp}^{2}\right)-\mathrm{H}$ 键的氟化可由简单的丙酮
肟醚导向实现(Eq. 12), 而更具挑战性的 $\mathrm{C}\left(\mathrm{sp}^{3}\right)-\mathrm{H}$ 键氟 化则需要丙酮酰胺衍生的双齿导向基团的协助(Eq. 13). 雷尼镍催化还原或六羰钼介导均可断裂 $\mathrm{N}-\mathrm{O}$ 键，脱 除导向基团得到简单醇化合物，因此该策略为氟代醇化 合物的制备提供了一种简洁通用的方法. 动力学同位素 效应(KIE)及氛代实验证明该反应经历了 $\mathrm{Pd}(\mathrm{II}) / \mathrm{Pd}(\mathrm{IV})$ 催化循环过程. 与 Scheme 2 描述的反应机理类似, 异丙 醇在丙酮酰胺衍生的双齿导向基团辅助下可形成 $\operatorname{Pd}(\mathrm{II})$ 中间体 8 , 其与 NFSI 氧化加成得到 $\operatorname{Pd}(\mathrm{IV})$ 中间体，随后 发生还原消除生成氟化产物并完成催化循环.
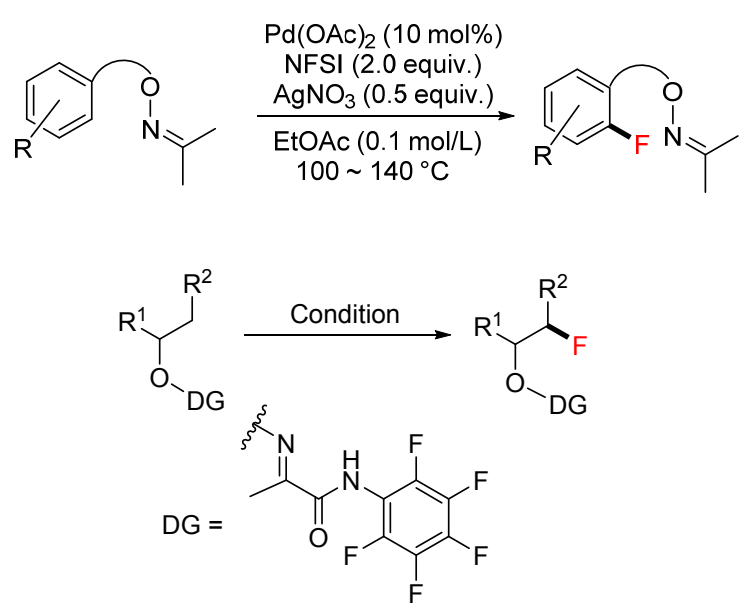

Condition a: $\mathrm{Pd}(\mathrm{OAc})_{2}(10 \mathrm{~mol} \%), \mathrm{NFSI}(2.0$ equiv.), $\mathrm{PhCl}$ Condition b: $\mathrm{Pd}(\mathrm{OAc})_{2}$ (10 mol\%), NFSI (2.0 equiv), $\mathrm{Ag}_{2} \mathrm{CO}_{3}$ (2.0 equiv.), $L$-leucine (10 $\mathrm{mol} \%), \mathrm{PhCl}$

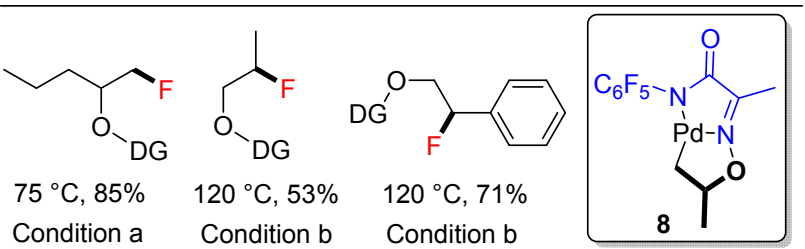

2020 年，余金权团队 ${ }^{[26]}$ 报道了脂肪胺 $\gamma$ 位的 $\mathrm{C}\left(\mathrm{sp}^{3}\right)-\mathrm{H}$ 氟化反应(Eq. 14). 该方法可实现不同官能团 取代的环已基和线性脂肪胺 $\gamma$-甲基和亚甲基上的 $\mathrm{C}-\mathrm{H}$ 键氟化. 值得注意的是该反应无需额外引入导向基团, 在瞬态导向基团 2-羟基烟醛辅助下即可实现，这大大提 高了反应的步骤经济性. NFSI 在反应中作为氟化试剂时 需添加银盐，原因是银盐可降低能量跨度，促进还原消 除步骤. 另外, 理论计算表明羟基吡啶作为配体辅助形 成了双金属过渡态，发生氧化加成反应. $N$-氟-2,4,6-三 甲基吡啶盐也能在该反应中作为优良的氟化试剂。

详细的催化循环如 Scheme 3 所示: 反应经历了 $\mathrm{Pd}(\mathrm{II}) / \mathrm{Pd}(\mathrm{IV})$ 催化循环过程. 脂肪胺首先与瞬态导向基 才 TDG 反应生成亚胺中间体 9, 在溶剂六氟异丙醇 $(\mathrm{HFIP})$ 中, $\mathrm{Pd}(\mathrm{OAc})_{2}$ 与 9 以及另一分子 $\mathbf{T D G}$ 作用, 经过 渡态 10 生成二价环钯中间体 $\mathbf{1 1}$. 在配体 $\mathbf{L}$ 和银盐促进 


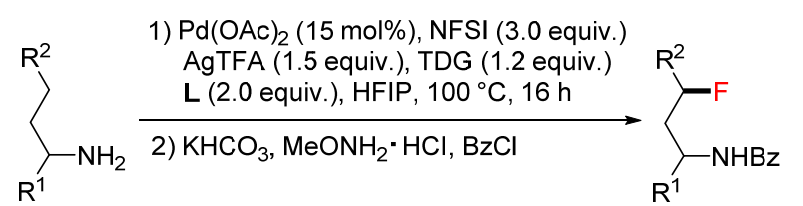<smiles>O=Cc1cccnc1O</smiles><smiles></smiles>

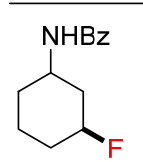

$70 \%$

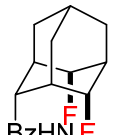

$51 \%$

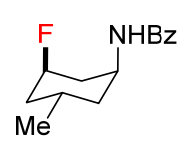

$66 \%$

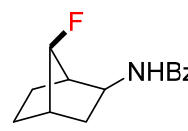

$74 \%$

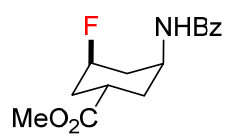

$55 \%$

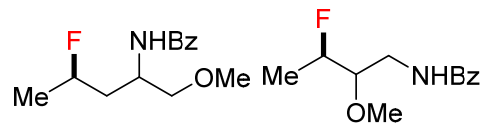

$39 \%(d . r=4.26: 1)$

$23 \%(d . r=10.1: 1)$
下, 二价环钯中间体 11 经过渡态 12 发生氧化加成, 生 成四价钯中间体 13. 中间体 13 转化为 14, 并发生还原 消除反应, 经过渡态 15 生成氟化产物 16, 伴随 $\operatorname{Pd}(\mathrm{OAc})_{2}$ 催化剂的生成, 完成催化循环. 16 原位水解得
到 $\gamma$-氟代胺化合物.

此外，其他氟化试剂也被用于过渡金属催化 $\mathrm{C}\left(\mathrm{sp}^{3}\right)-\mathrm{H}$ 氟化反应中: 如余金权 ${ }^{[27]}$ 、史炳锋 ${ }^{[28]}$ 和 $\mathrm{Ge}$ 课 题组 ${ }^{[29]}$ 以 Selectfluor 作为氟化试剂, 实现了烷基酰胺的 选择性 $\mathrm{C}\left(\mathrm{sp}^{3}\right)-\mathrm{H}$ 氟化; 此外, 余金权团队 ${ }^{[30]}$ 还以 $N$-氟2,4,6-三甲基吡啶盐作为有效的氟化试剂, 利用瞬态导 向策略实现苯甲醛衍生物苄基位对映选择性的 $\mathrm{C}\left(\mathrm{sp}^{3}\right)$ $\mathrm{H}$ 氟化; Sanford 团队 ${ }^{[31]}$ 则以 $\mathrm{AgF}$ 作为氟化试剂实现了 $\mathrm{Pd}$ 催化苄基位 $\mathrm{C}\left(\mathrm{sp}^{3}\right)-\mathrm{H}$ 氟化.

\section{$2 \mathrm{NFSI}$ 参与的 C一H 胺化反应}

在 $\mathrm{C}-\mathrm{H}$ 氟化反应中, 通常情况下反应底物通过形 成环金属中间体对 $\mathrm{C}-\mathrm{H}$ 键进行活化, 随后 NFSI 对环金 属中间体加成进而发生还原消除得到氟化产物. 但是在 $\mathrm{C}-\mathrm{H}$ 胺化反应中, 过渡金属催化 $\mathrm{C}-\mathrm{H}$ 键活化形成环 金属中间体后, NFSI 将其氧化成高价态金属中间体. 此 时，大位阻基团二苯磺酰亚胺基不易与金属中心结合， 更容易以亲核胺基化试剂的方式进攻环金属化合物. 另 一方面由于芳香环不容易被亲核试剂进攻，因此 NFSI 作为胺基化试剂，通过过渡金属催化 $\mathrm{C}-\mathrm{H}$ 键活化的策 略来构建 $\mathrm{C}\left(\mathrm{sp}^{2}\right)-\mathrm{N}$ 键的方法很少. 通常情况下 NFSI

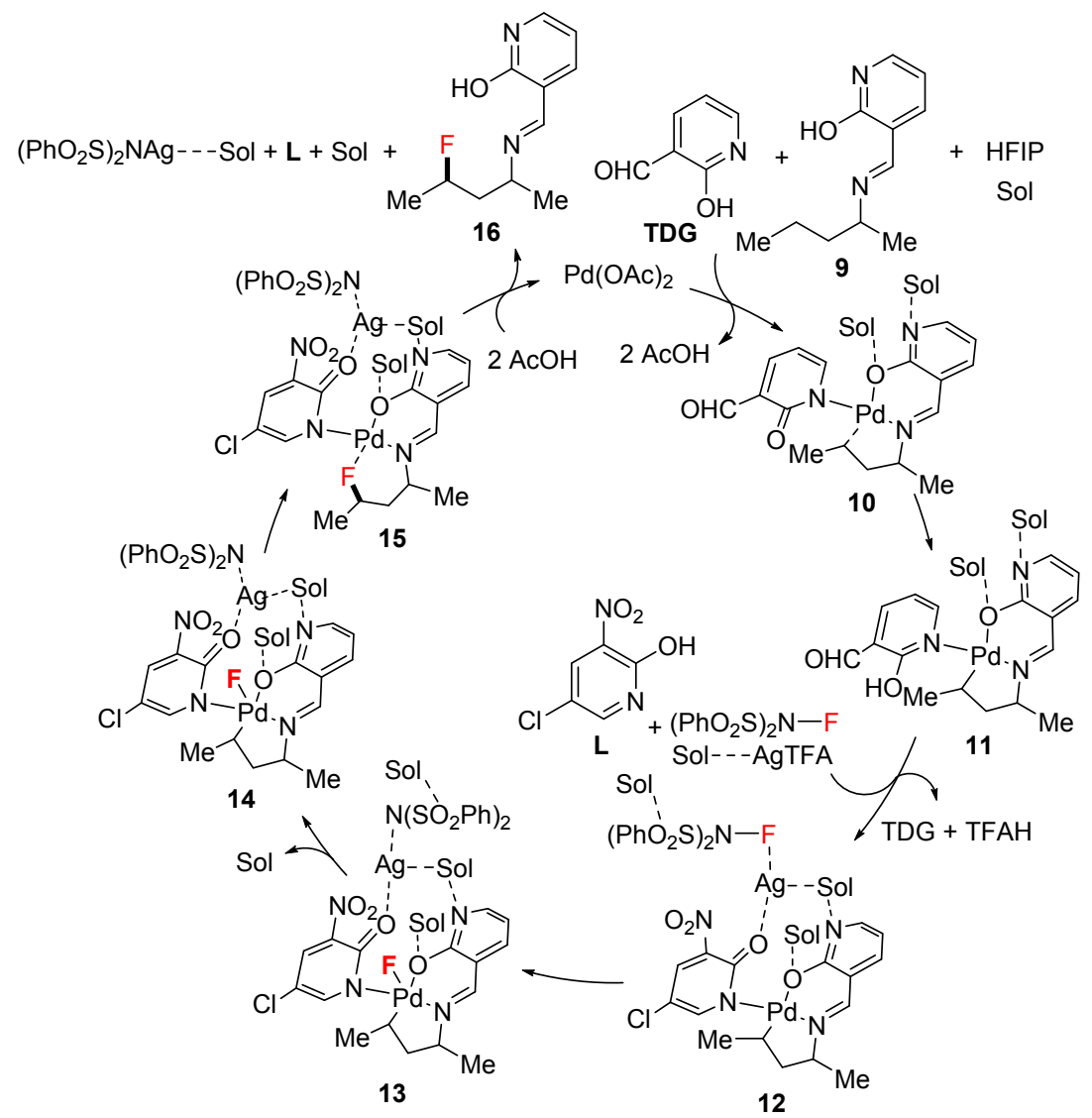

图式 3 瞬态导向辅助钯催化 $\mathrm{C}\left(s p^{3}\right)-\mathrm{H}$ 氟化催化循环

Scheme 3 Catalytic cycle for transient directing group-assisted Pd-catalyzed C-H fluorination 
对芳香烃 $\mathrm{C}\left(\mathrm{sp}^{2}\right)-\mathrm{H}$ 胺基化的方法往往是通过自由基过 程实现 ${ }^{[32]}$. 在本文中我们只聚焦 NFSI 参与的过渡金属 催化 $\mathrm{C}-\mathrm{H}$ 键活化构建 $\mathrm{C}-\mathrm{N}$ 键的方法, 对自由基机理 实现的芳香烃 $\mathrm{C}\left(s p^{2}\right)-\mathrm{H}$ 胺基化的方法将不展开讨论.

2013 年, 李朝军和杨罗等 ${ }^{[33]}$ 通过铑催化活化含氮 杂环邻位 $\mathrm{C}-\mathrm{H}$ 键, 在 NFSI 存在下实现了邻位 $\mathrm{C}-\mathrm{H}$ 胺 化反应(Eq. 15). 反应除铑催化剂外, 无需额外的氧化剂 或添加剂, 仅需催化量的碳酸铯作为碱即可实现. 反应 具有高选择性、高官能团兼容性等优点. 对于间位有官 能团取代的底物, 反应发生在空间位阻较小的一侧.

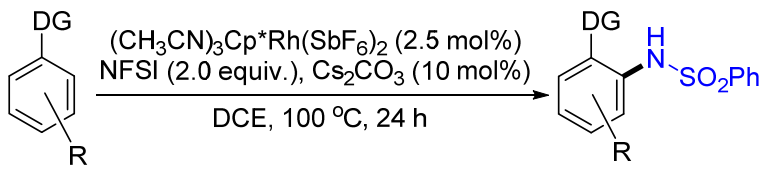<smiles>O=S(=O)(Nc1ccccc1-c1ccccc1)c1ccccc1</smiles>

$81 \%$<smiles>Cc1ccc(NS(=O)(=O)c2ccccc2)c(-c2ccccn2)c1</smiles>

$76 \%$<smiles>O=S(=O)(Nc1ccccc1-n1cccn1)Oc1ccccc1</smiles>

$32 \%$

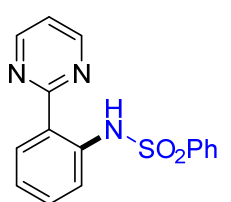

$58 \%$
作者推测反应机理可能如 Scheme 4 所示: 首先在 碳酸铯协助下, 含氮杂环导向 Rh(III)催化剂活化邻位 C - $\mathrm{H}$ 键生成环铑中间体 19. 中间体 19 与 NFSI 中的氟原 子配位生成中间体 20 , 随后, 中间体 20 发生金属转移 得到不稳定的中间体 21. 在空间位阻的影响以及底物 17 和 $\mathrm{CsHCO}_{3}$ 的共同作用下，中间体 21 的氟原子转移 到磺酰基上，进而脱去苯磺酰氟副产物 23 生成胺基化 产物 22, 同时得到活性铑物种 18 , 完成反应循环.

2019 年, Sharma 等 ${ }^{[34]}$ 报道了铑催化的喹啉氮氧化 合物 $\mathrm{C} 8$ 位的 $\mathrm{C}-\mathrm{H}$ 活化构建 $\mathrm{C}-\mathrm{Br}$ 键和 $\mathrm{C}-\mathrm{N}$ 键的方 法. 反应在 $N$-溴代琥珀酰亚胺(NBS)存在下发生溴化反 应，而 NFSI 或对甲苯磺酰叠氮则可作为胺基源用于 $\mathrm{C}-\mathrm{N}$ 键的构建(Eq. 16). 氧原子配位铑催化剂诱导活化 $\mathrm{C}(8)-\mathrm{H}$ 键形成的环铑化合物 24 是该反应的关键中间 体. 作者在无 NBS 或胺基源试剂存在下合成得到了该 中间体, 其作为催化剂同样可实现喹啉氮氧化合物 C8 位的 $\mathrm{C}-\mathrm{H}$ 官能团化反应(Eq. 17), 这也为反应机理的研 究提供了一定的佐证.

2012 年, Alvarez 和 Muniz 等 ${ }^{[35]}$ 发展了钯催化活化 $\mathrm{C}\left(\mathrm{sp}^{3}\right)-\mathrm{H}$ 键构建 $\mathrm{C}-\mathrm{N}$ 键的策略( $\mathrm{Eq}$. 18). 反应以 8-甲 基喹啉衍生物作为原料, 双(六氟乙酰丙酮)钯 $[\mathrm{Pd}(\mathrm{hf}-$

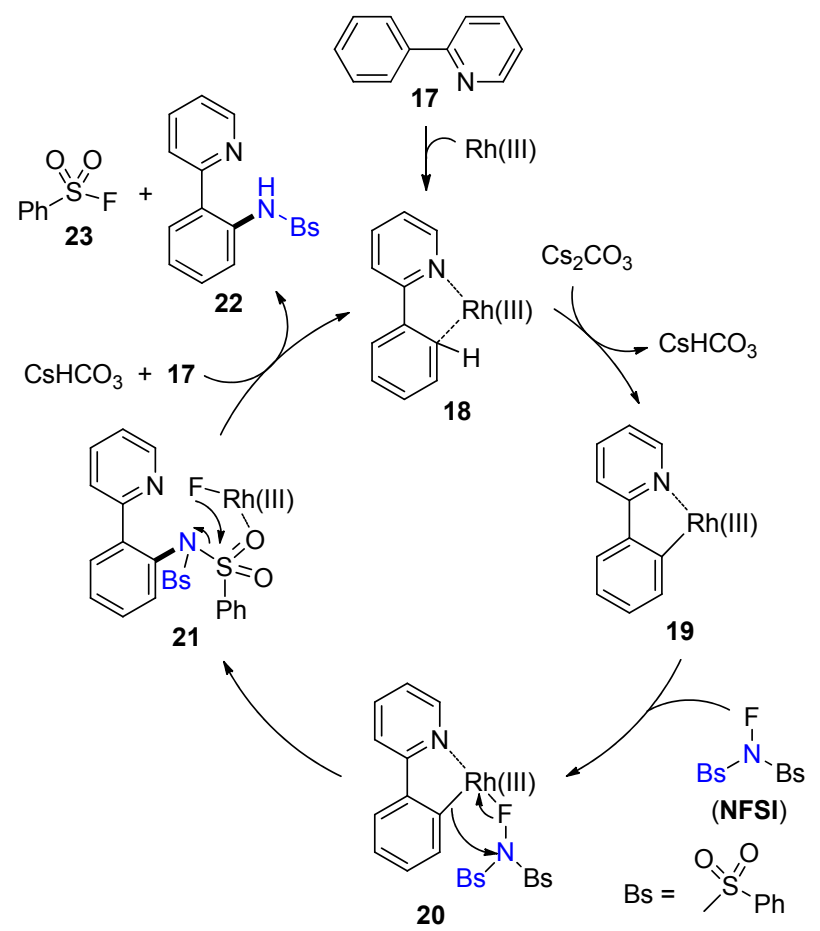

图式 4 铑催化 $\mathrm{C}-\mathrm{H}$ 胺基化机理图

Scheme 4 Mechanism for Rh-catalyzed $\mathrm{C}-\mathrm{H}$ amination
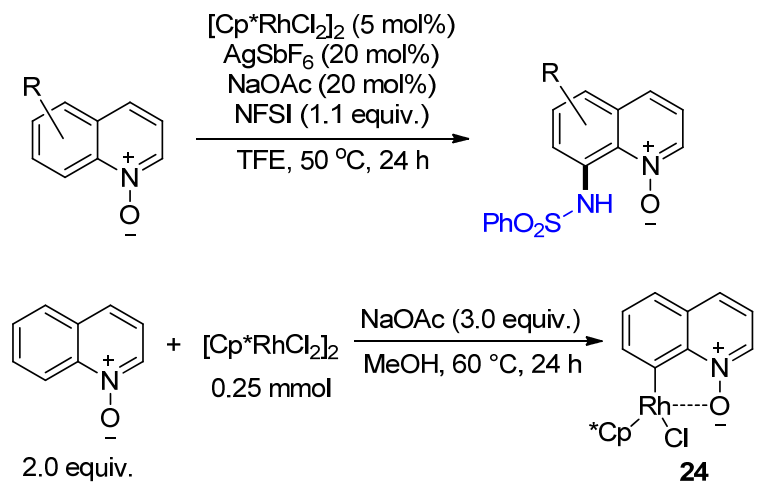

$\mathrm{acac})_{2}$ ] 作为催化剂, NFSI 作为胺化试剂，得到 8-甲基位 二苯磺酰亚胺基化产物, 其在酸性条件下可脱除苯磺酰 基得到 8-(胺甲基)喹啉衍生物. 2-叔丁基吡啶也可以在 该催化体系下实现甲基位上的 $\mathrm{C}\left(\mathrm{sp}^{3}\right)-\mathrm{H}$ 选择性单胺基 化反应(Eq. 19). 另外，甲氧基也可以作为定位基团，实 现邻甲基茴香醚衍生物苄基位的 $\mathrm{C}\left(\mathrm{sp}^{3}\right)-\mathrm{H}$ 胺基化(Eq. 20).

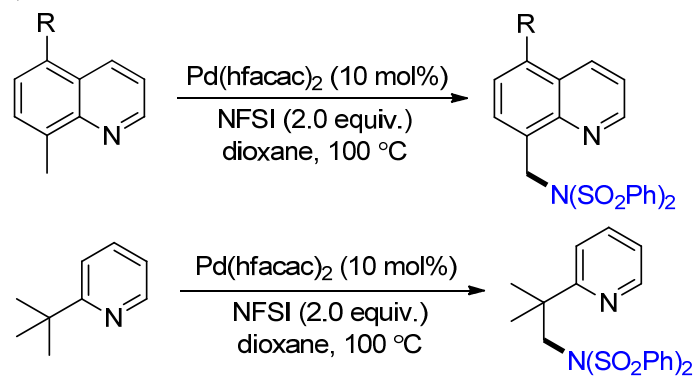




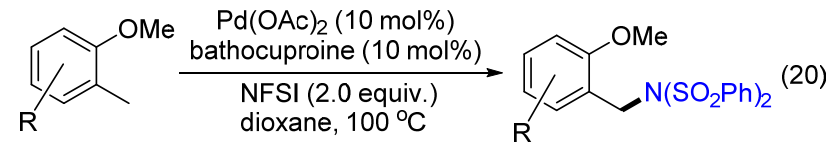

作者通过对照实验及理论计算推测该反应经历了 $\operatorname{Pd}(\mathrm{II}) / \mathrm{Pd}(\mathrm{IV})$ 的催化循环过程. 首先吡啶环与钯催化剂 配位，活化 $\mathrm{C}\left(\mathrm{sp}^{3}\right)-\mathrm{H}$ 键形成二价环钯中间体 26, 随后 26 被 NFSI 氧化形成四价钯中间体 27 并得到胺负离子. 胺负离子对四价钯中间体 27 亲核进攻得到 28 , 最后质 子解离生成胺化产物 29, 同时重新生成活性钯催化剂, 完成催化循环(Scheme 5). 值得注意的是, 催化循环过 程中生成的六氟乙酰丙酮(H-hfacac)对催化剂的重新生 成具有重要的作用, 作者使用钯化合物 26 作为反应催 化剂时, 在标准条件下只能以很低的收率得到胺化产 物, 但是六氟乙酰丙酮的添加可以将胺化产物的收率提 高到 92\% (Scheme 5).

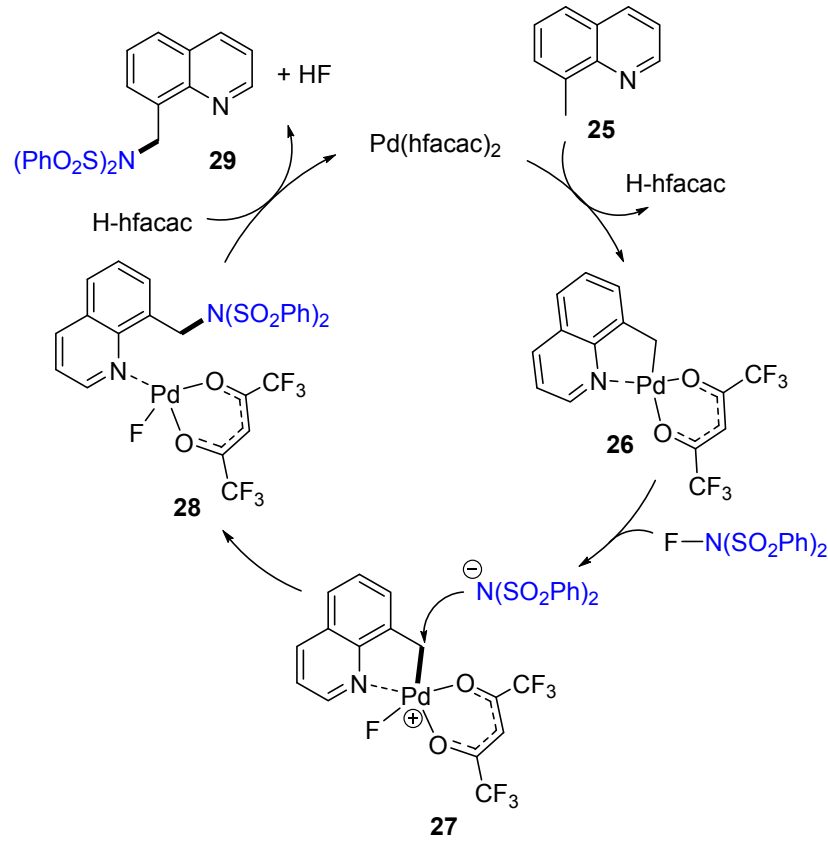

图式 $5 \mathrm{Pd}$ 催化 $\mathrm{C}\left(\mathrm{sp}^{3}\right)-\mathrm{H}$ 胺化反应机理图

Scheme 5 Mechanism for Pd-catalyzed $\mathrm{C}\left(\mathrm{sp}^{3}\right)-\mathrm{H}$ amination

2017 年, 刘鹏课题组 ${ }^{[36]}$ 研究了喹啉醛肟作为双齿 导向基团辅助钯催化的分子间 $\mathrm{C}\left(\mathrm{sp}^{3}\right)-\mathrm{H}$ 胺化反应( $\mathrm{Eq}$. $21)$. 该反应可选择性地实现醇衍生物 $\beta$ 位 $\mathrm{C}\left(\mathrm{sp}^{3}\right)-\mathrm{H}$ 胺 化, NFSI 既作为胺基源，同时在反应中也扮演氧化剂的 作用. 反应具有很好的官能团兼容性, 一级醇、二级醇 和三级醇均可顺利进行反应, 但三级醇的活性较低 (31d). 当 $\beta$ 位同时存在甲基和亚甲基时反应选择性发生 在甲基上的 $\mathrm{C}\left(\mathrm{sp}^{3}\right)-\mathrm{H}$ 键(31a), 另外 $\beta$ 位同时存在两个 甲基时, 以胺化 (32b) 产物为主, 并生成少量双胺化产物 (32b'). 导向基团在钯碳加氢条件下可脱除得到 1,2-胺 基醇衍生物，大大提高了该策略的实用价值. 同一时间,
刘刚课题组 ${ }^{[37]}$ 也报道了类似工作，通过对不同含氮杂 环导向基团的篎选，发现当使用喹啉醛肟作为导向基团 时胺化反应效果最佳(Eq. 22).

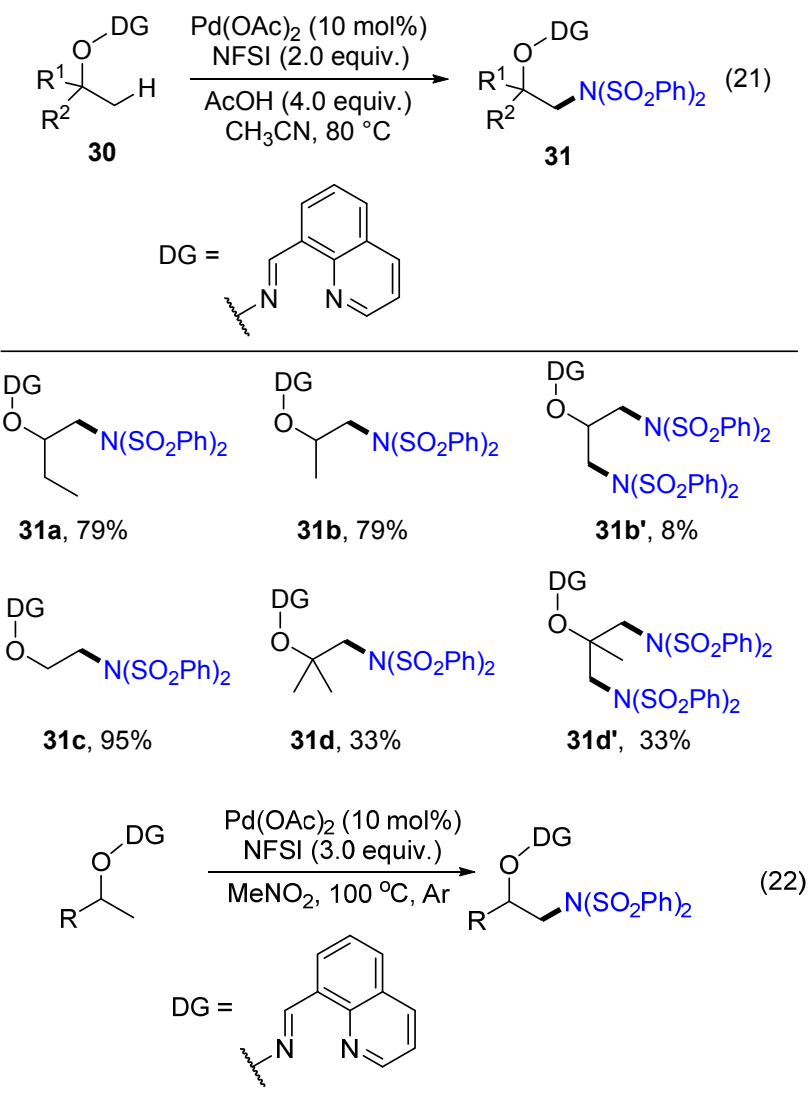

上述反应可能的催化循环起始于喹啉酫肜双齿导 向基团与过渡金属钯的配位. 中间体 32 脱去一分子酸 实现 $\beta$ 位 $\mathrm{C}\left(\mathrm{sp}^{3}\right)-\mathrm{H}$ 键的活化, 并形成二价环钯中间体 33. 随后中间体 33 与 NFSI 发生氧化加成形成四价钯中 间体 34, 其经过还原消除得到二价钯中间体 35, 最后配 体解离得到 $\beta$ 位 $\mathrm{C}\left(\mathrm{sp}^{3}\right)-\mathrm{H}$ 胺化产物 31 (Scheme 6).

大部分情况下，导向基团协助过渡金属催化 $\mathrm{C}-\mathrm{H}$ 键活化的策略往往只能实现导向基团邻位 $\mathrm{C}-\mathrm{H}$ 键的官 能团化. 2011 年, 张前团队 ${ }^{[38]}$ 报道了一种钯催化的远程 $\mathrm{C}-\mathrm{H}$ 胺基化的方法. NFSI 作为胺化试剂, 实现了乙酰 苯胺衍生物对位的 $\mathrm{C}\left(\mathrm{sp}^{2}\right)-\mathrm{H}$ 胺基化反应(Eq. 23). 值得 注意的是, 只有当乙酰苯胺底物的邻位有烷氧基官能团 取代时，反应才能顺利进行. 另外，当乙酰苯胺的对位 有取代基时，胺化反应则发生在乙酰氨基的邻位.

作者推测反应经历了 $\mathrm{Pd}(0) / \mathrm{Pd}(\mathrm{II})$ 的催化循环过程. $\operatorname{Pd}(0)$ 与 NFSI 直接发生氧化加成生成二价钯物种 $\mathbf{3 9}, \mathbf{3 9}$ 与底物乙酰苯胺发生亲电钯化反应, 经过渡态 $\mathbf{4 0}$ 生成 螺环型的二价环钯中间体 41. 在酰胺和氧正基团的共 同作用下，胺负离子选择性进攻中间体 41 乙酰胺基的 对位形成二价钯中间体 42. 最后重芳构化生成对位胺 


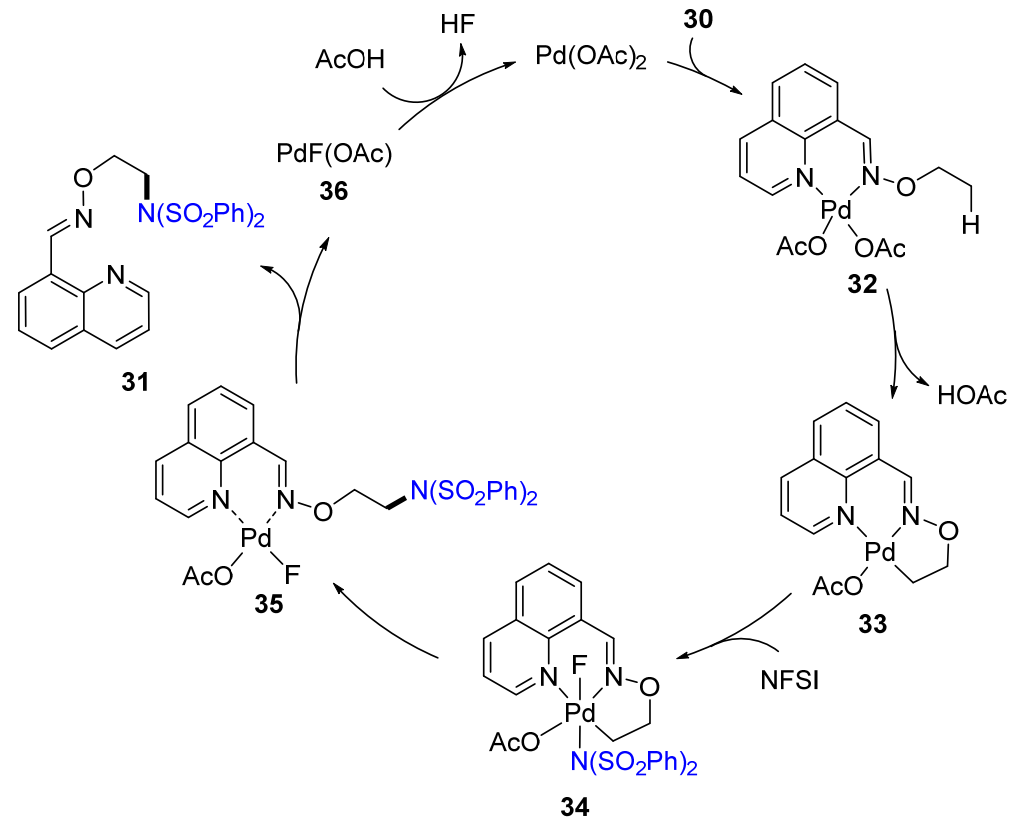

图式 6 双齿导向基辅助钯催化醇 $\beta-\mathrm{C}\left(\mathrm{sp}^{3}\right)-\mathrm{H}$ 胺化的催化循环图

Scheme 6 Catalytic cycle for bidentate directing group-assisted Pd-catalyzed $\beta$-C $\left(\mathrm{sp}^{3}\right)-\mathrm{H}$ amination

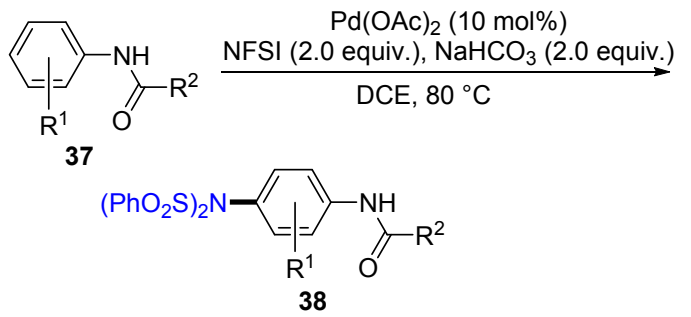

(23)<smiles>COc1cc(N(CC(C)C)OCC(C)C)ccc1NC(=O)C(C)(C)C</smiles><smiles>CCOc1cc(N=COS(=O)(=O)OC(C)C)ccc1NC(=O)C(C)(C)C</smiles><smiles>CCCCOCCN(C(=O)O[Na])c1ccc(N2CCCC2=O)c(OC)c1</smiles><smiles>CC(=O)Nc1ccc(-c2ccccc2)cc1NS(=O)(=O)O</smiles>

$62 \%$

基化的产物 38, 同时重新生成零价钯催化剂, 完成催化 循环(Scheme 7).

上述策略不仅可以实现远程的 $\mathrm{C}\left(\mathrm{sp}^{2}\right)-\mathrm{H}$ 胺化反应， 还可以对酰基苯胺衍生物远程 $\mathrm{C}\left(\mathrm{sp}^{3}\right)-\mathrm{H}$ 键进行定位得 到对位茮基胺化的产物(Eq. 24) ${ }^{[39]}$. 张前和张景萍等认 为反应可能经历了 $\mathrm{Pd}(\mathrm{II}) / \mathrm{Pd}(\mathrm{IV})$ 的催化循环过程. 理论 计算结合实验证明 ${ }^{[40]}$, 反应底物首先在 NFSI 存在的条 件下与 $\mathrm{Pd}(\mathrm{OAc})_{2}$ 作用生成螺环型的四价环钯中间体 $\mathbf{4 5}$. 45 通过金属协同去氢(CMD)过程和还原消除得到中间 体 46 和二苯磺酰亚胺, 同时生成二价钯物种 47. 另一 方面, 中间体 46 与二苯磺酰亚胺发生亲核加成生成茮 基位胺化的产物 44 (Scheme 8). 作者指出水作为质

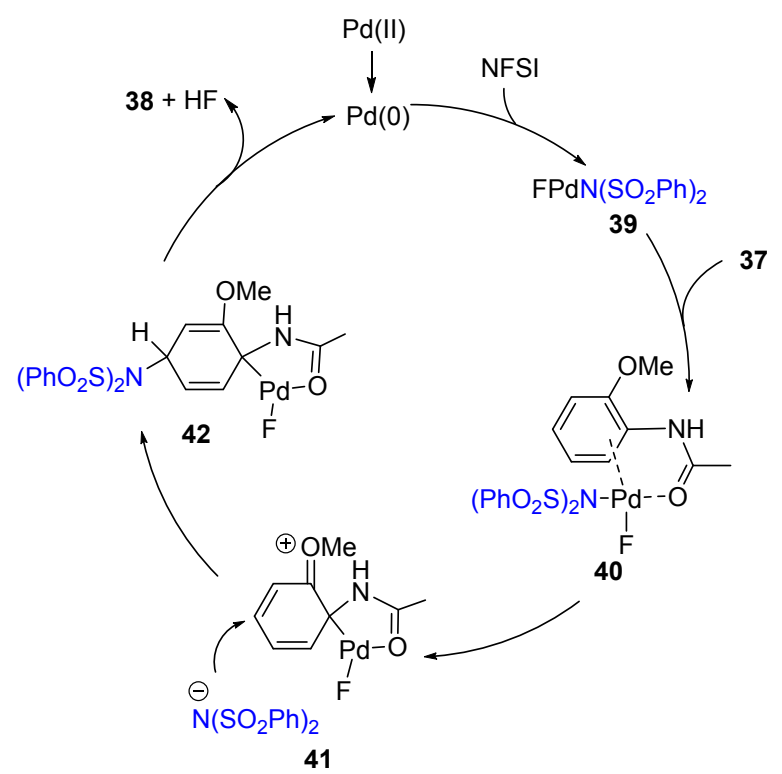

图式 $7 \mathrm{Pd}$ 催化远程 $\mathrm{C}\left(s p^{2}\right)-\mathrm{H}$ 胺化的催化循环图 Scheme 7 Catalytic cycle for Pd-catalyzed remote $\mathrm{C}\left(s p^{2}\right)-\mathrm{H}$ amination.

子转移的桥梁, 在 CMD 和还原消除过程中都起到了重 要的作用, 而 NFSI 在反应中不仅作为胺基源, 同时也 扮演氧化剂的作用(将二价态钯氧化成四价态钯). 尽管 从上述两种钯催化远程 $\mathrm{C}\left(\mathrm{sp}^{2}\right)-\mathrm{H}$ 和 $\mathrm{C}\left(\mathrm{sp}^{3}\right)-\mathrm{H}$ 胺基化 反应的机理中可以看出, 过渡金属并未实现 $\mathrm{C}-\mathrm{H}$ 键真 正的断裂活化，但是该策略具备独特的创新性和反应选 择性. 因此我们认为在此讨论将有助于加深读者对过渡 金属催化 $\mathrm{C}-\mathrm{H}$ 胺化反应构建的理解. 


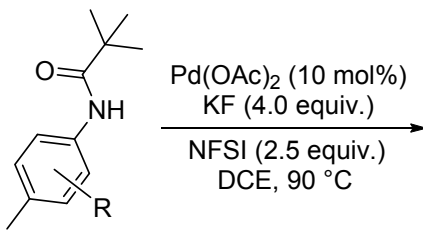

43

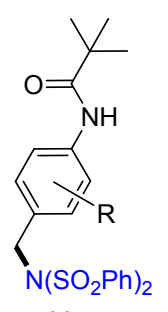

44
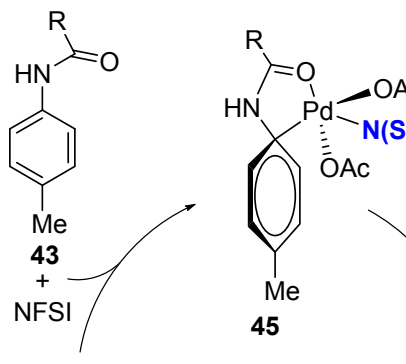

$\mathrm{Pd}(\mathrm{OAc})_{2}$
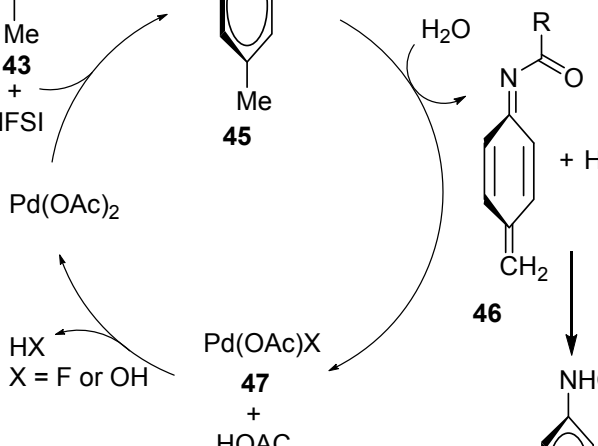

$+\mathrm{HN}\left(\mathrm{SO}_{2} \mathrm{Ph}\right)_{2}$

$\left(\mathrm{PhO}_{2} \mathrm{~S}\right)_{2} \mathrm{~N}$

44

图式 $8 \mathrm{Pd}$ 催化远程 $\mathrm{C}\left(\mathrm{sp}^{3}\right)-\mathrm{H}$ 胺化的催化循环图 Scheme 8 Catalytic cycle for Pd-catalyzed remote $C\left(\mathrm{sp}^{3}\right)-\mathrm{H}$ amination

\section{3 总结与展望}

NFSI 兼具氟原子及含氮官能团, 在过渡金属催化 的 $\mathrm{C}-\mathrm{H}$ 活化反应中, 既可以构建 $\mathrm{C}-\mathrm{F}$ 键, 也可以用来 形成 $\mathrm{C}-\mathrm{N}$ 键, 从而为含氟和含氮化合物的制备提供新 的合成策略. 在 $\mathrm{C}-\mathrm{H}$ 氟化的方法中, NFSI 往往是通过 对 $\mathrm{C}-\mathrm{H}$ 活化形成的环金属化合物进行氧化加成实现氟 化作用, 这与绝大多数的过渡金属催化 $\mathrm{C}-\mathrm{H}$ 键活化反 应机理一致, 因此 $\mathrm{C}\left(\mathrm{sp}^{2}\right)-\mathrm{H}$ 键的氟化反应更容易发生. 但是, 在 $\mathrm{C}-\mathrm{H}$ 胺化的方法中, 二苯磺酰亚胺的位阻较 大，不易与金属中心结合，因此通常以亲核进攻的方式 与环金属化合物作用. 由于 $\mathrm{C}\left(\mathrm{sp}^{3}\right)-\mathrm{Pd}$ 键比 $\mathrm{C}\left(\mathrm{sp}^{2}\right)-\mathrm{Pd}$ 键更易被亲核试剂进攻, 因此 NFSI 参与的 $\mathrm{C}-\mathrm{H}$ 活化反 应中, $\mathrm{C}\left(\mathrm{sp}^{3}\right)-\mathrm{H}$ 键的胺化反应更容易进行.

综上所述，由于 NFSI 参与的过渡金属催化 $\mathrm{C}-\mathrm{H}$ 键活化反应中，氟化和胺化反应具有不同的反应机制， 使 NFSI 可选择性地构建 $\mathrm{C}\left(\mathrm{sp}^{2}\right)-\mathrm{F}$ 键和 $\mathrm{C}\left(\mathrm{sp}^{3}\right)-\mathrm{N}$ 键. 另外 NFSI 还可以通过自由基过程构建 $\mathrm{C}\left(\mathrm{sp}^{3}\right)-\mathrm{F}$ 键和 $\mathrm{C}\left(\mathrm{sp}^{2}\right)-\mathrm{N}$ 键, 二者恰好形成互补. 但是, 与 NFSI 参与
的其他众多反应类型相比, NFSI 用于 $\mathrm{C}-\mathrm{H}$ 键活化的方 法报道仍然较少. 因此，设计使 NFSI 选择性实现 $\mathrm{C}-\mathrm{H}$ 氟化或胺化的催化体系仍具有很强的吸引力. 其次, 利 用廉价金属替代贵金属催化剂, 发展条件温和的 $\mathrm{C}-\mathrm{H}$ 氟化和胺化反应仍是该领域研究的长期目标. 最后, 通 过对 NFSI 在 C-H 键活化反应中的机理进行深入研究, 开发专一多能, 可实现多种官能团化的新型偶联试剂, 对 $\mathrm{C}-\mathrm{H}$ 键活化领域的发展将具有重要意义.

\section{References}

[1] (a) Liao, G.; Wu, Y.-J.; Shi, B.-F. Acta Chim. Sinica 2020, 78, 289 (in Chinese)

(廖港，吴勇杰，史炳锋，化学学报，2020，78，289.)

(b) Zhao, Q.; Meng, G.; Nolan, S. P.; Szostak, M. Chem. Rev. 2020, 120, 1981.

(c) Mao, P.; Zhu, J.; Yuan, J.; Yang, L.; Xiao, Y.; Zhang, C. Chin. J. Org. Chem. 2019, 39, 1529 (in Chinese).

(毛璞, 朱军亮, 袁金伟, 杨亮茹, 肖咏梅, 张长森, 有机化学, 2019, 39, 1529.)

(d) Rej, S.; Chatani, N. Angew. Chem. Int. Ed. 2019, 58, 8304

(e) Duarah, G.; Kaishap, P. P.; Begum, T.; Gogoi, S. Adv. Synth. Catal. 2019, 361, 654.

(f) Luo, F. Chin. J. Org. Chem. 2019, 39, 3084 (in Chinese). (罗飞华，有机化学, 2019, 39, 3084.)

(g) Chu, J. C. K.; Rovis, T. Angew. Chem. Int. Ed. 2018, 57, 62.

(h) Wang, S.; Yan, F.; Wang, L.; Zhu, L. Chin. J. Org. Chem. 2018, 38, 291 (in Chinese)

(汪珊, 严沣, 汪连生, 朱否, 有机化学, 2018, 38, 291.)

[2] (a) Friis, S. D.; Johansson, M. J.; Ackermann, L. Nat. Chem. 2020, 12,511 .

(b) Gruss, H.; Sewald, N. Chem.-Eur. J. 2020, 26, 5328

(c) Zhang, Y.; Shen, S.; Fang, H.; Xu, T. Org. Lett. 2020, 22, 1244.

(d) Ren, Q.; Nie, B.; Zhang, Y.; Zhang, J. Chin. J. Org. Chem. 2018, 38, 2465 (in Chinese).

(任青云, 聂崤, 张英俊, 张雯, 有机化学, 2018, 38, 2465.)

[3] (a) Felpin, F.-X.; Sengupta, S. Chem. Soc. Rev. 2019, 48, 1150. (b) Xu, P.; Duan, X. Chin. J. Org. Chem. 2019, 39, 3315 (in Chinese).

(徐鹏, 段新红, 有机化学, 2019, 39, 3315.)

(c) Nareddy, P.; Jordan, F.; Szostak, M. ACS Cat. 2017, 7, 5721.

[4] (a) Das, R.; Kapur, M. Asian J. Org. Chem. 2018, 7, 1524.

(b) Luo, J.; Xu, X.; Zhao, Y.; Liang, H. Chin. J. Org. Chem. 2017, 37, 2873 (in Chinese).

(骆钧飞, 徐星, 赵延超, 梁洪泽, 有机化学, 2017, 37, 2873.)

(c) Liao, G.; Shi, B.-F. Acta Chim. Sinica 2015, 73, 1283 (in Chinese).

(廖港, 史炳锋, 化学学报, 2015, 73, 1283.)

[5] (a) Szpera, R.; Moseley, D. F. J.; Smith, L. B.; Sterling, A. J. Gouverneur, V. Angew. Chem. Int. Ed. 2019, 58, 14824.

(b) He, J.; Lou, S.; Xu, D. Chin. J. Org. Chem. 2016, 36, 1218 (in Chinese).

(何将旗, 娄绍杰, 许丹倩, 有机化学, 2016, 36, 1218.)

(c) Liang, T.; Neumann, C. N.; Ritter, T. Angew. Chem. Int. Ed. 2013, 52, 8214.

[6] (a) Engle, K. M.; Mei, T.-S.; Wang, X.; Yu, J.-Q. Angew. Chem. Int. Ed. 2011, 50, 1478.

(b) Wang, X.; Leow, D.; Yu, J.-Q. J. Am. Chem. Soc. 2011, 133, 13864 .

(c) Ball, N. D.; Gary, J. B.; Ye, Y.; Sanford, M. S. J. Am. Chem. Soc. 2011, 133, 7577.

[7] (a) Rueda-Becerril, M.; Sazepin, C. C.; Leung, J. C. T.; Okbinoglu, T.; Kennepohl, P.; Paquin, J.-F.; Sammis, G. M. J. Am. Chem. Soc. 2012, 134, 4026 . 
(b) Halperin, S. D.; Fan, H.; Chang, S.; Martin, R. E.; Britton, R. Angew. Chem. Int. Ed. 2014, 53, 4690.

(c) Sibi, M. P.; Landais, Y. Angew. Chem. Int. Ed. 2013, 52, 3570

[8] (a) Sibbald, P. A.; Rosewall, C. F.; Swartz, R. D.; Michael, F. E. J. Am. Chem. Soc. 2009, 131, 15945.

(b) Weng, S.-S.; Hsieh, K.-Y.; Zeng, Z.-J.; Zhang, J.-W. Tetrahedron Lett. 2017, 58, 670.

[9] (a) Qiu, S.; Xu, T.; Zhou, J.; Guo, Y.; Liu, G. J. Am. Chem. Soc. 2010, 132, 2856.

(b) Zhang, H.; Song, Y.; Zhao, J.; Zhang, J.; Zhang, Q. Angew. Chem. Int. Ed. 2014, 53, 11079 .

[10] (a) Zhang, H.; Pu, W.; Xiong, T.; Li, Y.; Zhou, X.; Sun, K.; Liu, Q.; Zhang, Q. Angew. Chem. Int. Ed. 2013, 52, 2529.

(b) Wang, D.; Wang, F.; Chen, P.; Lin, Z.; Liu, G. Angew. Chem. Int. Ed. 2017, 56, 2054.

[11] (a) Zhang, B.; Studer, A. Org. Lett. 2014, 16, 1790.

(b) Lei, B.; Wang, X.; Ma, L.; Li, Y.; Li, Z. Org. Biomol. Chem. 2018, 16, 3109 .

[12] (a) Wang, D.; Wu, L.; Wang, F.; Wan, X.; Chen, P.; Lin, Z.; Liu, G. J. Am. Chem. Soc. 2017, 139, 6811.

(b) Zheng, G.; Sun, J.; Liu, Y.; Yang, S.; Li, Y.; Sun, H.; Zhang, Q. J. Org. Chem. 2017, 82, 12813.

[13] (a) Ni, Z.; Zhang, Q.; Xiong, T.; Zheng, Y.; Li, Y.; Zhang, H.; Zhang, J.; Liu, Q. Angew. Chem. Int. Ed. 2012, 51, 1244

(b) Zhang, X.; Wu, R.; Liu, W.; Qian, D.-W.; Yang, J.; Jiang, P.; Zheng, Q.-Z. Org. Biomol. Chem. 2016, 14, 4789.

(c) Bao, F.; Cao, Y.; Liu, W.; Zhu, J. RSC Adv. 2019, 9, 27892.

[14] Lou, S. J.; Xu, D. Q.; Xia, A. B.; Wang, Y. F.; Liu, Y. K.; Du, X. H.; Xu, Z. Y. Chem. Commun. 2013, 49, 6218.

[15] Lou, S. J.; Xu, D. Q.; Xu, Z. Y. Angew. Chem.; Int. Ed. 2014, 53, 10330.

[16] Lou, S.-J.; Chen, Q.; Wang, Y.-F.; Xu, D.-Q.; Du, X.-H.; He, J.-Q.; Mao, Y.-J. Xu, Z.-Y. ACS Catal. 2015, 5, 2846

[17] Ning, X.-Q.; Lou, S.-J.; Mao, Y.-J.; Xu, Z.-Y.; Xu, D.-Q. Org. Lett. 2018, 20, 2445.

[18] Testa, C.; Gigot, E.; Genc, S.; Decreau, R.; Roger, J.; Hierso, J.-C. Angew. Chem. Int. Ed. 2016, 55, 5555 .

[19] Testa, C.; Roger, J.; Scheib, S.; Fleurat-Lessard, P.; Hierso, J. C. Adv. Synth. Catal. 2015, 357, 2913.

[20] Ding, Q. P.; Ye, C. Q.; Pu, S. Z.; Cao, B. P. Tetrahedron 2014, 70, 409.

[21] Chen, C.; Wang, C.; Zhang, J.; and Zhao, Y. J. Org. Chem. 2015, 80,942 .

[22] Gutierrez, D. A.; Lee, W.-C. C.; Shen, Y.; Li, J. J. Tetrahedron Lett. 2016, $57,5372$.
[23] Lee, J. B.; Kang, M. E.; Kim, J.; Lee, C. Y.; Kee, J.-M.; Park, K. Myung, J.-U.; Hong, S. Y. Chem. Commun.; 2017, 53, 10394

[24] Zhu, Q.; Ji, D.; Liang, T.; Wang, X.; Xu, Y. Org. Lett. 2015, 17, 3798.

[25] Mao, Y.-J.; Lou, S.-J.; Hao, H.-Y.; Xu, D.-Q. Angew. Chem.; Int Ed. 2018, 57, 14085.

[26] Chen, Y.-Q.; Singh, S.; Wu, Y.; Wang, Z.; Hao, W.; Verma, P.; Qiao, J. X.; Sunoj, R. B.; Yu, J.-Q. J. Am. Chem. Soc. 2020, 142, 9966.

[27] Zhu, R. Y.; Tanaka, K.; Li, G. C.; He, J.; Fu, H. Y.; Li, S. H.; Yu, J.-Q. J. Am. Chem. Soc. 2015, 137, 7067.

[28] Zhang, Q.; Yin, X. S.; Chen, K.; Zhang, S. Q.; Shi, B.-F. J. Am. Chem. Soc. 2015, 137, 8219.

[29] Miao, J.; Yang, K.; Kurek, M.; Ge, H. Org. Lett. 2015, 17, 3738.

[30] Park, H.; Verma, P.; Hong, K.; Yu, J.-Q. Nat. Chem. 2018, 10, 755

[31] McMurtrey, K. B.; Racowski, J. M.; Sanford, M. S. Org. Lett. 2012, 14, 4094.

[32] (a) Lu, S.; Tian, L.-L.; Cui, T.-W.; Zhu, Y.-S.; Zhu, X.; Hao, X.-Q.; Song, M.-P. J. Org. Chem. 2018, 83, 13991.

(b) Haines, B. E.; Kawakami, T.; Kuwata, K.; Murakami, K.; Itami, K.; Musaev, D. G. Chem. Sci. 2017, 8. 988.

(c) Yin, Y.; Xie, J.; Huang, F.-Q.; Qi, L.-W.; Zhang, B. Adv. Synth. Catal. 2017, 359, 1037.

(d) Barve, B. D.; Wu, Y.-C.; El-Shazly, M.; Korinek, M.; Cheng, Y.-B.; Wang, J.-J.; Chang, F.-R. Tetrahedron. 2015, 71, 2290.

(e) Kawakami, T.; Murakami, K.; Itami, K. J. Am. Chem. Soc. 2015, 137, 2460 .

(f) Wang, S.; Ni, Z.; Huang, X.; Wang, J.; Pan, Y. Org. Lett. 2014, 16,5648 .

(g) Wang, X.; Lei, B.; Ma, L.; Jiao, H.; Xing, W.; Chen, J.; Li, Z. Adv. Synth. Catal. 2013, 359, 4284

[33] Tang, R.-J.; Luo, C.-P.; Yang, L.; Li, C.-J. Adv. Synth. Catal. 2013, $355,869$.

[34] Dhiman, A. K.; Gupta, S. S.; Sharma, R.; Kumar, R.; Sharma, U. J. Org. Chem. 2019, 84, 12871.

[35] Iglesias, A.; Alvarez, R.; de Lera, A. R.; Muniz, K. Angew. Chem. Int. Ed. 2012, 51, 2225.

[36] Jin, L.; Zeng, X.; Li, S.; Hong, X.; Qiu, G. Liu. P. Chem. Commun. 2017, 53, 3986.

[37] Dong, Y.; Liu, G. J. Org. Chem. 2017, 82, 3864

[38] Sun, K.; Li, Y.; Xiong, T.; Zhang, J.; Zhang, Q. J. Am. Chem. Soc. 2011, 133, 1694.

[39] Zhou, Y.-F.; Zhu, J.-M.; Li, B.; Zhang, Y.; Feng, J.; Hall, A.; Shi, J.-Y.; Zhu, W.-L. Org. Lett. 2016, 18, 3803

[40] Zheng, Y.; Xiong, T.; Lv, Y.; Zhang, J.; Zhang, Q. Org. Biomol. Chem. 2013, 11, 7923. 